\title{
Fluoroscopy-Assisted Percutaneous Endoscopic Gastrostomy (F-PEG)-Single-Center Experience and Outcome
}

\author{
Avinash Bhat Balekuduru $\quad$ Shruti Sagar Bongu' Narendra Babu Mandalapu ${ }^{1}$ \\ Gajendra Ramachandraiah ${ }^{1}$ Vinit Kumar Khemka ${ }^{1}$ Satyaprakash Bonthala Subbaraj ${ }^{1}$ \\ ${ }^{1}$ Department of Gastroenterology, M.S. Ramaiah Hospitals, \\ Bangalore, Karnataka, India \\ Address for correspondence Avinash Bhat Balekuduru, DM, \\ Department of Gastroenterology, M.S. Ramaiah Hospitals, \\ Bangalore 560054, India (e-mail: avinashbalekuduru@gmail.com).
}

J Digest Endosc:2020;11:193-200

\begin{abstract}
Keywords

- percutaneous endoscopic gastrostomy

- fluoroscopy

- complications

- F-PEG
\end{abstract}

Background Gastrointestinal route is considered for feeding in subjects who are unable to swallow, either as a temporary or permanent option. Percutaneous endoscopic gastrostomy (PEG) is the preferred mode for long-term enteral nutrition. The aim was to study the outcome of protocol-based PEG placement in a tertiary medical center.

Materials and Methods All the patients who underwent PEG placement between January 2017 and December 2019 were included in the retrospective study. Study variables were entered into a uniform structured proforma. The procedure was done by two people using Ponsky-Gauderer pull-technique. Fluoroscopy guidance was considered for placement in special situations. Post-procedure, all the patients were regularly followed as per protocol to evaluate for adverse events.

Results One hundred and eighteen patients with placement of PEG were included in the study. The mean age of the patients was $49.6 \pm 7$ years with $67.8 \%$ males. The most common indication of PEG was inability to swallow associated with head injury (43, 36.4\%), followed by carcinoma esophagus $(35,29.8 \%)$ and stroke $(24,20.3 \%)$. Fluoroscopy was useful in 38 (32\%) for PEG site identification. Tube dislodgement $(16,13.5 \%)$ and aspiration pneumonia $(20,16.9 \%)$ were the common adverse events. Age more than 60 years and dysphagia in neurologic disorders accounted for more than $60 \%$ of adverse events. Forty-nine (42\%) of the PEG patients expired due to either primary illness or due to sepsis after a median time of 139 days (range: 32-288 days). There was no difference in the survival in patients with or without PEG-related complications $(p=0.74)$.

Conclusions Fluoroscopy assistance helps in accurate PEG placement in one third. Age $>60$ years and dysphagia in neurologic disorders were independent risk factors associated with PEG tube complications.

\section{Introduction}

Percutaneous endoscopic gastrostomy (PEG) was first reported by Gauderer et al. ${ }^{1}$ For enteral nutrition, PEG gained preference over the already established surgical or radiological gastrostomy due to superior outcome both in terms of morbidity and mortality. ${ }^{2-4}$ For long-term nutrition in neurological dysphagia, PEG is preferred over nasogastric (NG) tube due to aesthetic appeal, avoidance of uncomfortable oropharyngeal symptoms, less chance of oropharyngeal
DOI https://doi.org/

$10.1055 / \mathrm{s}-0040-1716580$

ISSN 0976-5042.
License terms

()(1) $\Theta \circledast$ 
candidiasis and respiratory infections, averting esophageal ulcer and stricture, and reduced risk of clogging or displacement of tube. ${ }^{5-8}$ Also, overnight feeding is safer with a PEG tube. ${ }^{9}$

In contrast to PEG, the placement of NG tube is relatively easy at the bedside by trained medical staff. The accuracy of NG tube position can be easily confirmed by auscultation over the upper abdomen on air insufflation through the tube or by aspiration of gastric contents and occasionally by radiographic examination. ${ }^{10}$

Although PEG placement is safe, it has both early and late complications like bleeding, peritonitis, bowel obstruction or perforation, and abscess at the operative site. ${ }^{7,11,12}$ The reported PEG-related morbidity and mortality are 9-17\% and $0-10 \%$, respectively. ${ }^{3,13}$ The American Society of Gastrointestinal Endoscopy recommends PEG insertion in patients who are expected to survive longer than 1 month after the procedure. ${ }^{14}$

The use of fluoroscopy at PEG tube placement (F-PEG) is infrequently reported. ${ }^{15}$ In patients with ventriculoperitoneal shunts, fluoroscopy assists in identifying and avoiding it, and helps select a suitable site for PEG placement. Fluoroscopy is also useful in patients having altered upper gastrointestinal (GI) anatomy, kyphoscoliosis, gastric volvulus, or pseudoobstruction. It can occasionally help in confirming accidental colonic or small bowel injury during puncture and also aids in detection of any significant pneumo-peritoneum at the time of procedure. ${ }^{16}$

PEG remains an underutilized procedure in India, mainly due to lack of inclination or awareness among the treating physicians. ${ }^{17}$ The aim of our study was to study the outcome of protocol-based PEG placement in a tertiary medical center.

\section{Materials and Methods}

Data was collected retrospectively for all the patients who underwent a PEG tube placement between January 2017 and December 2019 at our hospital. The study was approved by Institute Ethical Committee.

The baseline data points captured included age, gender, comorbidities, indications, and contraindications for PEG. Also, regular follow-up data was maintained (especially looking for adverse events associated with PEG), including its removal. The patients underwent the procedure after required informed consent. All the procedures were performed by the same endoscopic team under supervised anesthesia.

\section{Inclusion Criteria}

Patients presenting with neurological diseases having reduced level of consciousness (e.g., cerebrovascular disease, motor neuron disease, head injury), cancer of head and neck or esophagus, and gastric volvulus were included. All patients were on enteral feeds through a NG prior to PEG tube insertion. In gastric volvulus, fixation of anterior stomach wall to the abdominal wall was the primary aim to prevent recurrence. In patients on dual antiplatelets (aspirin and clopidogrel), clopidogrel was stopped 7 days before the procedure while continuing aspirin. Patients on warfarin were changed over to low molecular weight heparin for 5 days before the procedure. The last dose of heparin was given 12 hours before procedure and restarted after 6 hours, post-procedure.

\section{Exclusion Criteria}

Following are the exclusion criteria: Coagulopathy (International Normalized Ratio $>1.5$, partial thromboplastin time $>50$ seconds, platelets $<50,000 / \mathrm{mm}^{3}$ ), hemodynamic instability, sepsis, gross ascites, peritonitis, peritoneal carcinomatosis, history of total gastrectomy, gastric outlet obstruction, and lack of informed consent for the procedure. Those with documented fever or significant leucocytosis were excluded.

\section{Technique}

All the patients received betadine oral gargle on the night before procedure. After overnight fasting, oral cleansing was performed in the morning on the day of procedure. Prophylactic antibiotic was given before the procedure (1 gm of ceftriaxone/vancomycin).

The procedure was performed in an endoscopy room having fluoroscopy facility. PEG procedure was done by two people using Ponsky-Gauderer pull-technique using standard flexible endoscope (GIF-Q 150/H 180-Olympus, Tokyo, Japan) and 24Fr gastrostomy tube (Wilson-Cook, Winston-Salem, North Carolina, United States). ${ }^{18}$ At endoscopy, luminal contents were cleared including from hypopharynx (-Fig. 1).

A balloon replacement tube (BRT) was used for PEG exchange when required. The BRT tube was inserted at previous gastrostomy site and balloon was inflated to the capacity with prefilled syringes.
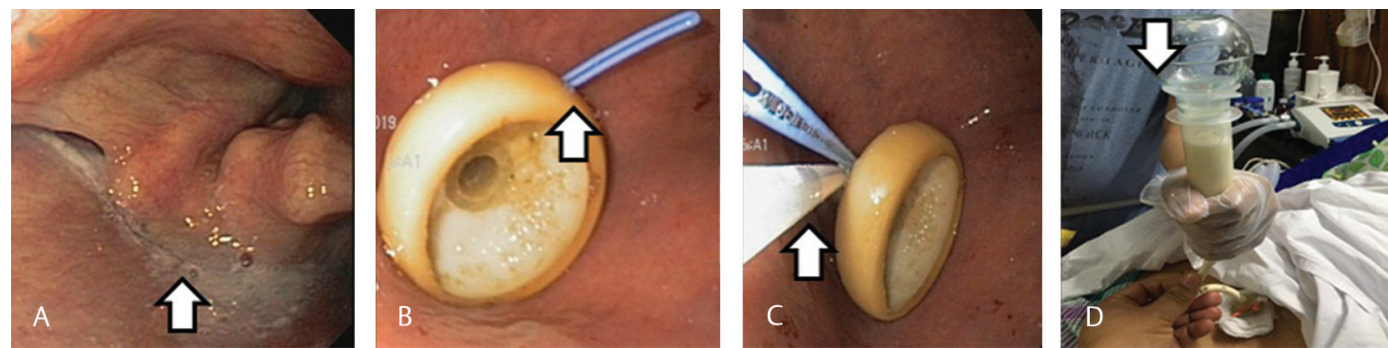

Fig. 1 (A) Pooling of secretions in the posterior pharyngeal wall; (B) Guidewire (GW) wire passed along side of existing percutaneous endoscopic gastrostomy (PEG) tube that was held by snare as in image (C) for PEG exchange. In image (D), an Asepto pump was being used for home-based blenderized feeds. 


\section{Post-PEG Care}

Patients were kept nil orally after PEG procedure for 8 hours. Prior to PEG feeding, the tube site was inspected and free rotation of tube and the external bolster was confirmed. Tube feeding was started with 100 to $200 \mathrm{~mL}$ of clear liquids, after ensuring no abdominal pain, vomiting, or tube leakage as per protocol. ${ }^{19}$ After 4 hours, PEG feeds were initiated in boluses of $250 \mathrm{~mL}$ in 20 minutes for every 4 hours. Flushing with $20 \mathrm{~mL}$ of water was done after every feeding. At discharge, the patients' family were instructed about feeding, and made aware of potential PEG-related complications (mentioned below) and follow-up (either telephonically or physically) as per clinical requirement.

Aspiration pneumonia was defined by new development of chest symptoms (cough, purulent sputum, or fever after PEG) with changes in chest radiograph and/or oxygen saturation and/or witnessed aspiration. ${ }^{20}$ Dislodgement was defined as complete inadvertent pulling out of gastrostomy tube. ${ }^{20}$ Buried bumper syndrome (BBS) was considered when the inner bumper migrates alongside the stoma tract out of the gastric lumen and can be anywhere between the stomach mucosa and the surface of the skin. BBS was suspected when there was increased leakage around the PEG tube, along with pain or resistance to PEG feeding. ${ }^{21}$ Peristomal infections (PSI) were defined as having at least two of the following: peristomal erythema, induration, purulent discharge with supportive laboratory evidence of infection/positive culture report. ${ }^{22,23}$ Positive culture growth but no clinical signs were regarded as colonization and were not included under PSI. Bleeding from the PEG tract was defined by bleeding from any side of the PEG tract that can present as external wound bleeding, subcutaneous hematoma at PEG site, melena or other forms of GI bleeding confirmed to be from PEG site on endoscopy and/or drop in hemoglobin concentration. ${ }^{24}$ PEG tube obstruction was identified by inability to pass feed or flush water down the tube with no visible sign of tube kinking. ${ }^{24}$

The patients that recovered from primary illness and resumed oral intake had their PEG tube removed.

\section{Statistical Analysis}

Data were reported as mean \pm standard deviation) for continuous variables and frequencies (percentages) for categorical variables. All analyses were performed using the SPSS statistical analysis software version 17.0 (SPSS Inc., Chicago, Illinois, United States). Survival was assessed using KaplanMeier curve after comparing median survival time using Koch's regression model. All tests were two-sided and statistical significance was defined as a $p$-value less than 0.05 .

\section{Results}

Two hundred and seventy-five PEG procedures were performed during the study period. The flowchart depicts the final number included in the study ( - Fig. 2). The demographic data of the study group is presented in - Table $\mathbf{1}$. The mean age of the patients was $49.6 \pm 7$ years (range: $10-95$ years). Majority of the patients were males (80,67.8\%). Inability to swallow associated with head injury was the most common

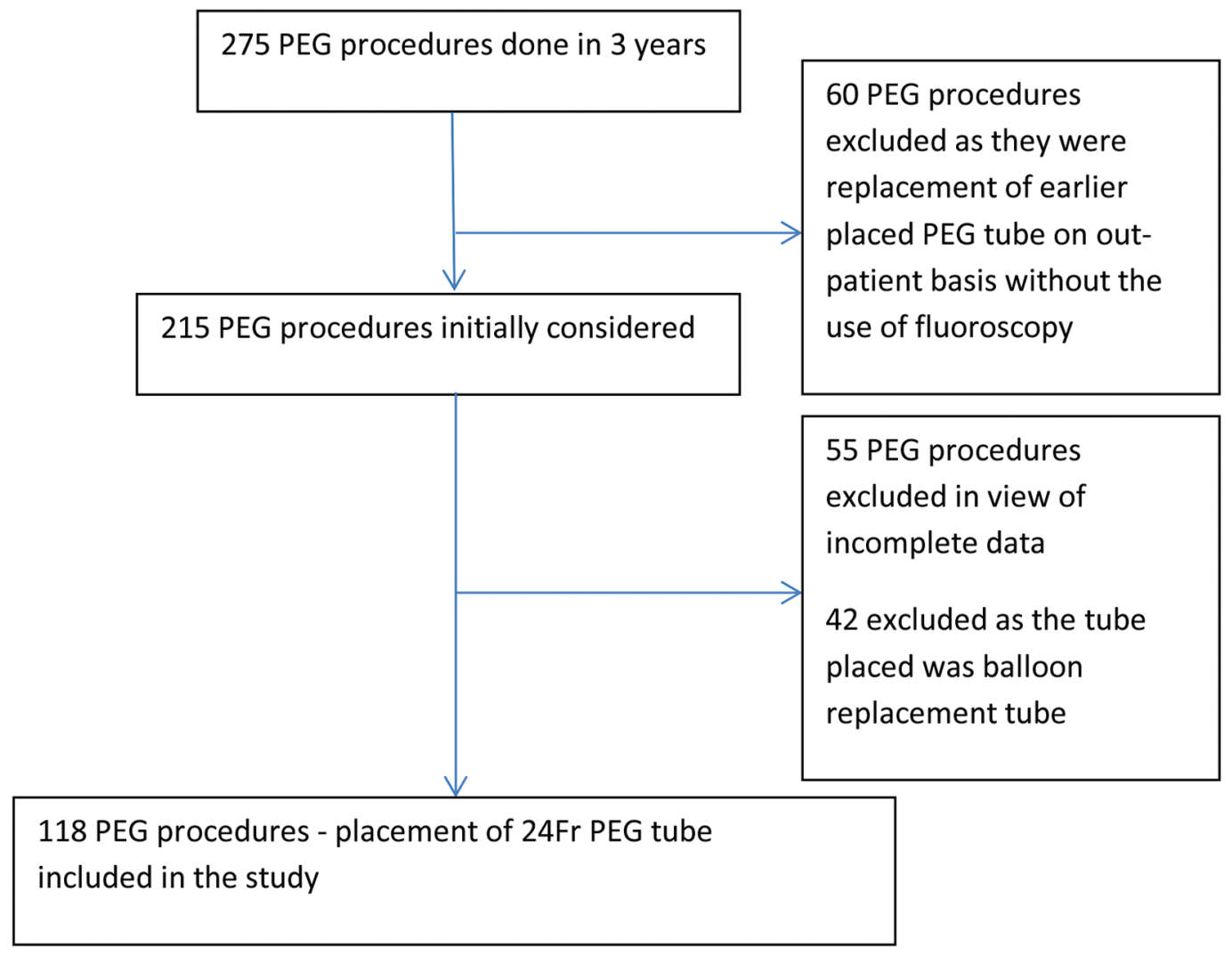

Fig. 2 Study flowchart showing patient recruitment in the study. PEG, percutaneous endoscopic gastrostomy. 
Table 1 Demography of the subjects who underwent PEG procedure

\begin{tabular}{|c|c|c|}
\hline SI No & $\begin{array}{l}\text { Variable } \\
n=118(100 \%)\end{array}$ & $\begin{array}{l}\text { Frequency/ } \\
\text { Number (\%) }\end{array}$ \\
\hline 1 & Male: Female & $\begin{array}{l}80(67.8 \%): \\
38(32.2 \%)\end{array}$ \\
\hline 2 & $\begin{array}{l}\text { Age: Mean } \pm \text { SD (range) } \\
\text { in years }\end{array}$ & $49.6 \pm 7$ y $(10-95$ y) \\
\hline \multirow[t]{6}{*}{3} & Indication for PEG procedure & \\
\hline & RTA & $43(36.4 \%)$ \\
\hline & CE & $35(29.8 \%)$ \\
\hline & CVA & $24(20.3 \%)$ \\
\hline & MND & $15(12.7 \%)$ \\
\hline & Gastric volvulus & $1(0.8 \%)$ \\
\hline \multirow[t]{5}{*}{4} & Comorbidities & \\
\hline & Hypertension & $49(42 \%)$ \\
\hline & Diabetes mellitus & $41(35 \%)$ \\
\hline & Heart disease & $17(15 \%)$ \\
\hline & Seizure disorder & $13(11 \%)$ \\
\hline \multirow[t]{4}{*}{5} & Concurrent medicines & \\
\hline & Aspirin & $48(41 \%)$ \\
\hline & Clopidogrel & $39(38 \%)$ \\
\hline & Warfarin & $16(14 \%)$ \\
\hline \multirow[t]{7}{*}{6} & PEG complications & $30(25.4 \%)$ \\
\hline & PEG site bleeding & $3(2.5 \%)$ \\
\hline & Aspiration pneumonia & $15(12.7 \%)$ \\
\hline & Buried bumper syndrome & $15(12.7 \%)$ \\
\hline & Peristomal infection & $13(11 \%)$ \\
\hline & Tube dislodgement & $16(13.5 \%)$ \\
\hline & Tube clogging & $9(7.6 \%)$ \\
\hline 7 & $\begin{array}{l}\text { Median follow-up with IQR in } \\
\text { days }\end{array}$ & 91 days (32-730 d) \\
\hline \multirow[t]{6}{*}{8} & Mortality at follow-up & $49(42 \%)$ \\
\hline & Primary disease & $29(59.2 \%)$ \\
\hline & Aspiration pneumonia & $12(24.5 \%)$ \\
\hline & Sepsis/septic shock & $8(16.3 \%)$ \\
\hline & On PEG feeds till last follow-up & $13(10 \%)$ \\
\hline & PEG tube removal at follow-up & $56(48 \%)$ \\
\hline
\end{tabular}

Abbreviations: BBS, buried bumper syndrome; $C E$, carcinoma esophagus; CVA, cerebrovascular accident; IQR, interquartile range; MND, motor neurone disease; PEG, percutaneous endoscopic gastrostomy; RTA, road traffic accident; SD, standard deviation.

indication $(43,36.4 \%)$ followed by carcinoma esophagus $(35,29.8 \%)$ and stroke $(24,20.3 \%)$.

In carcinoma esophagus ( $n=35)$, luminal dilatation with bougie was required in $25(71.4 \%)$ patients prior to PEG placement. Fifty-six (48\%) patients returned to oral feeding after radiotherapy and PEG tube was removed during the study period. Overall, 56 (48\%) patients had removal of PEG tube that occurred between 12 weeks and 1 year of the placement. A total of 83 (71\%) had comorbidities (-Table 1). PEG-related complications were observed in $30(25.4 \%)$ patients. The association of the complications with age, gender and the indication for PEG placement are described in - Table 2. There was no PEG tube-related mortality.

\section{PEG Site Identification and Fluoroscopy}

The endoscopic transillumination, finger indentation, and safe needle track techniques were used for PEG site selection. Fluoroscopy was utilized in 38 (32.2\%) patients of the total cases. In five cases, transillumination was not feasible due to thick abdominal wall. Ventriculoperitoneal shunt was present in 10 patients where fluoroscopy helped to map it out and avoid any accidental injury. In 15 cases of tube dislodgement and 8 cases of BBS, under fluoroscopy contrast injection with a cannula was used for assessing the previous tract patency and for PEG tube replacement ( - Fig. $\mathbf{3}$ ). In three cases of PEG site bleeding, the puncture was observed to be closer to the gastric wall. We assume that bleeding can be reduced if the puncture can be positioned away from the sides of stomach. The average dose of exposure in the use was $2-4 \mathrm{mSv}$ (4-8 spot images) in intermittent fluoroscopy.

\section{Tube Dislodgement}

Tube dislodgement (-Fig. 4) was the most common complication observed in $16(13.5 \%)$ patients. Age $>60$ years and dysphagia in neurologic disorders (DIN) were significantly associated with tube dislodgement $(p$-value $=0.001)$ (-Table 2). All dislodgements occurred after 4 weeks of PEG placement. In 12 cases, a $16 \mathrm{~F}$ or $18 \mathrm{~F}$ Foley catheter was placed as a temporary measure by a trained staff at home. Later, reinsertion of PEG was done. In other four cases, the event occurred during the hospital stay and a new PEG tube was reinserted.

\section{Aspiration Pneumonia}

Aspiration pneumonia was noted in $15(12.7 \%)$ patients with significant association with DIN ( $p$-value $=0.028$ ) (-Table 2). They were managed conservatively using standard procedures.

\section{Peristomal Infection)}

A total of 13 (11\%) cases had growth of organisms from PEG site (12 g-negative, $1 \mathrm{~g}$-positive organisms). Ten cases had purulent secretion with sterile cultures and were not included in PSI. They were managed successfully with regular dressing. The positive isolates included Klebsiella, ${ }^{6}$ Pseudomonas, ${ }^{8}$ Acinetobacter, ${ }^{1}$ and Staphylococcus aureus. ${ }^{1}$ They were managed with antibiotics and regular dressing without removal of PEG tube. There were positive cultures from other sites: blood $(n=7)$, urine $(n=19)$, and tracheostomy catheter $(n=1)$. Two patients had recurrent PEG site infection and required multiple courses of antibiotics (-Fig. 5). In none of the cases, the PEG site cultures matched with other site cultures, though they were done in the same admission. 
Table 2 Association of the complications with age, gender, and the indication for PEG placement

\begin{tabular}{|l|l|l|l|l|}
\hline $\begin{array}{l}\text { SI. } \\
\text { No }\end{array}$ & Complications $(\boldsymbol{n})$ & Variable & $\boldsymbol{n}(\%)$ & $\begin{array}{l}\boldsymbol{p} \text {-Value (Fisher's exact } \\
\text {-test value) }\end{array}$ \\
\hline 1 & $\begin{array}{l}\text { Aspiration pneumonia } \\
15(12.7 \%)\end{array}$ & $\begin{array}{l}\text { Age (y): }<60: \geq 60 \\
\text { Gender: Male: female } \\
\text { Indication: }\end{array}$ & $\begin{array}{l}9(60 \%): 6(40 \%) \\
10(66.7 \%): 5(33.3 \%) \\
1(6.6 \%): 14(93.3 \%)\end{array}$ & 0.774 \\
& & CE: DIN & 0.729 \\
& & Age (y): $<60: \geq 60$ & $3(23 \%): 10(77 \%)$ & 0.028 \\
\hline 2 & Peristomal infection & Gender: Male: female & $5(38 \%): 8(62 \%)$ & $0.04(0.07)$ \\
& $13(11 \%)$ & Indication: CE: DIN & $3(23 \%): 10(76.9 \%)$ & 0.001 \\
\hline 3 & BBS & Age (y): $<60: \geq 60$ & $3(20 \%): 12(80 \%)$ & 0.001 \\
& $15(12.7 \%)$ & Gender: Male: female & $6(40 \%): 9(60 \%)$ & $0.037(0.06)$ \\
\hline 4 & Tube dislodgement & Age (y): $<60: \geq 60$ & $2(13.3 \%): 13(86.7 \%)$ & 0.001 \\
\hline & $16(13.5 \%)$ & Gender: Male: female & $3(18.7 \%): 13(81.2 \%)$ & 0.001 \\
& & Indication: CE: DIN & $2(12.5 \%): 14(87.5 \%)$ & 0.983 \\
\hline
\end{tabular}

Abbreviations: BBS, buried bumper syndrome; CE, carcinoma esophagus; DIN, dysphagia in neurologic disorders; PEG, percutaneous endoscopic gastrostomy.
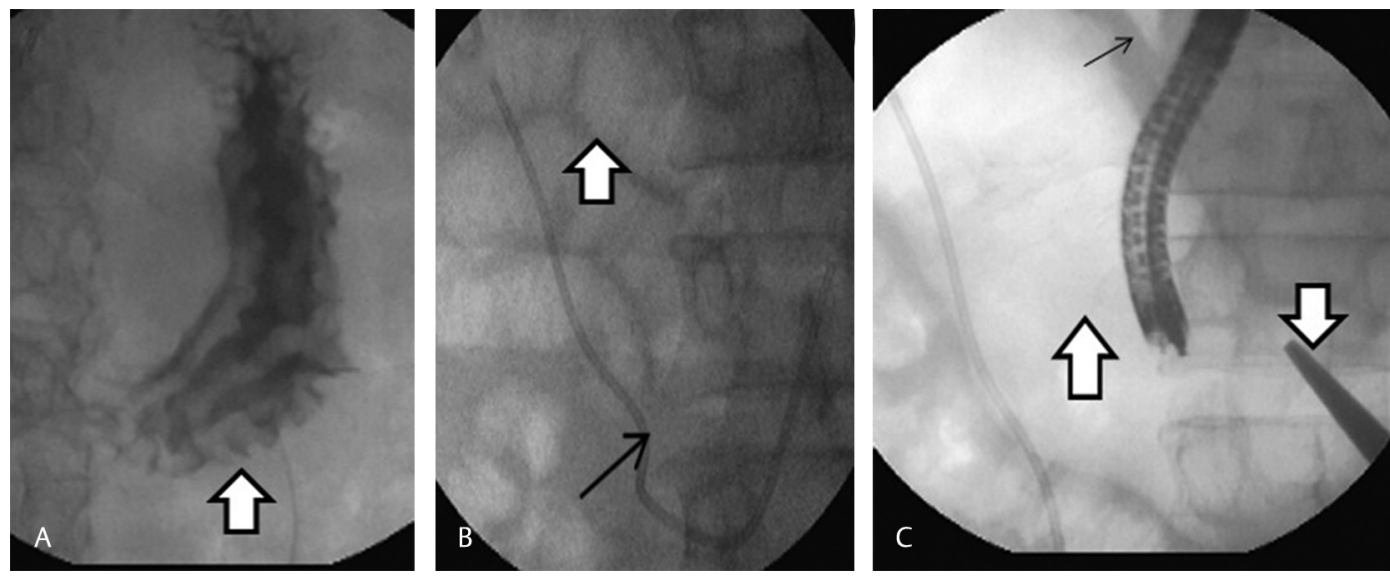

Fig. 3 Image (A) shows delineating stomach with contrast through a cannula in tube dislodgement to identify the patency of the tract or presence of any leak. In image (B), bold white arrow shows dilated small bowel loops and black arrow points to ventriculoperitoneal (VP) shunt. (C) Endoscope and Aerogastria with delineated walls in a case of VP shunt (bold white arrow). Black arrow points to left crus of diaphragm. Inverted arrow points to anticipated percutaneous endoscopic gastrostomy site away from small bowel and VP shunt tube.
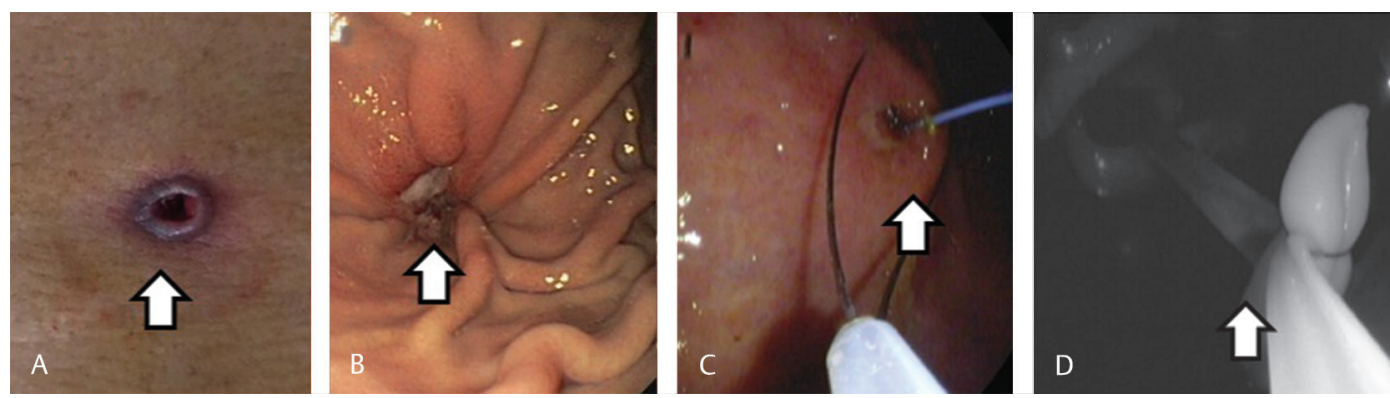

Fig. 4 (A) The abdominal end and (B) endoscopic side of the gastrostomy site in a case of percutaneous endoscopic gastrostomy (PEG) tube dislodgement. (C) GW was directly passed through gastrostomy site into the snare positioned at endoscopic end. (D) Same snare was used to hold the internal bumper to ease the intubation of scope at PEG placement.
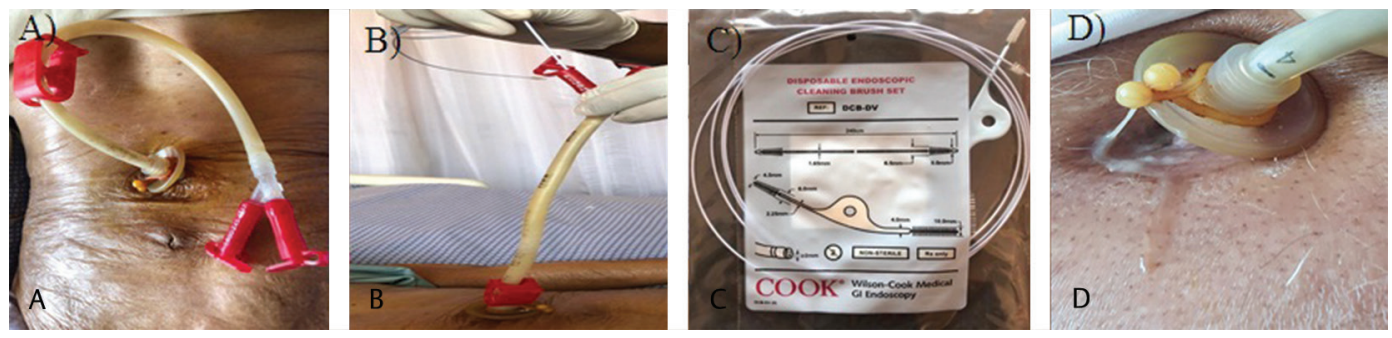

Fig. 5 (A) Percutaneous endoscopic gastrostomy (PEG) tube obstruction, (B) The technique to de-clogging with endoscopic cleaning brush as in images (C) and in (D) peristomal infection-discharge from PEG site. 


\section{Buried Bumper Syndrome}

All the $15(12.7 \%)$ cases of BBS presented with inability to feed or peristomal tightness. The urgent endoscopic assessment was done for suspected PEG tube malposition. PEG tube was removed by traction at the abdominal end and a new PEG tube was placed at the same site. Age $>60$ years and DIN were significantly associated with both BBS and PSI $(p$-value $=0.001)(-$ Table 2$)$

\section{PEG Site Bleeding}

Three patients presented with melena and peritubal bleeding in less than 7 days after PEG insertion (-Fig. 6). One patient required blood transfusion. All the bleeding complications happened in hospital and were on combination of clopidogrel and aspirin. All the patients required tightening of external bumper and endoscopic injection of diluted adrenaline at the PEG site. ${ }^{24}$

\section{Tube Obstruction}

Tube obstruction was noted in five cases (-Fig. 5). The PEG tube was cleaned with brush (Wilson Cook Medical GI Endoscopy,Cook, Ireland) and warm water, without the need for replacement.

\section{PEG Tube Removal}

PEG tube was removed in 56 (48\%) patients on their recovery (between 12 weeks and 1 year after PEG insertion) either by external traction removal or under direct endoscopic vision by snaring the inner bumper and cutting loose the external bumper for the removal. Endoscopic removal was performed in 23 carcinoma esophagus cases that also enabled to assess the disease response to radiotherapy.

\section{Survival}

Forty-nine (42\%) of the PEG patients expired due to either primary illness or due to sepsis (-Table 1). Aspiration pneumonia was the cause of death in 12 (24.5\%) of the patients. There was no mortality due to PEG tube procedure or PEG tube per se. There was no significant difference in median survival in patients having PEG-related complications ( $n=30 ; 208$ 191-224 days) compared with those without ( $n=88,214$ (28-233 days); $(p=0.740)$. The survival depended more on the primary disease than on the PEG-related complications (-Fig. 7).

\section{Discussion}

PEG is cost-effective, minimally invasive method of establishing prolonged enteral feeding ( $>4$ weeks), hydration, and medication administration for patients unable to sustain oral intake. ${ }^{25,26}$ Adequate appropriate enteral nutrition can benefit critically ill patients by reducing complication rate and hospital stay. ${ }^{27}$

The most common indication for PEG tube placement is dysphagia secondary to cerebrovascular accident (CVA), ${ }^{28}$ but in the present study, road traffic accident was the most common indication followed by carcinoma esophagus and CVA. This may be due to the referral pattern.

The technical success of PEG placement in the present study was $100 \%$. The PEG placement was performed in endoscopy room with fluoroscopy facility (F-PEG). The fluoroscopy provided the advantage in accurate selection of the site of skin puncture. ${ }^{29}$ In F-PEG, full gastric insufflation

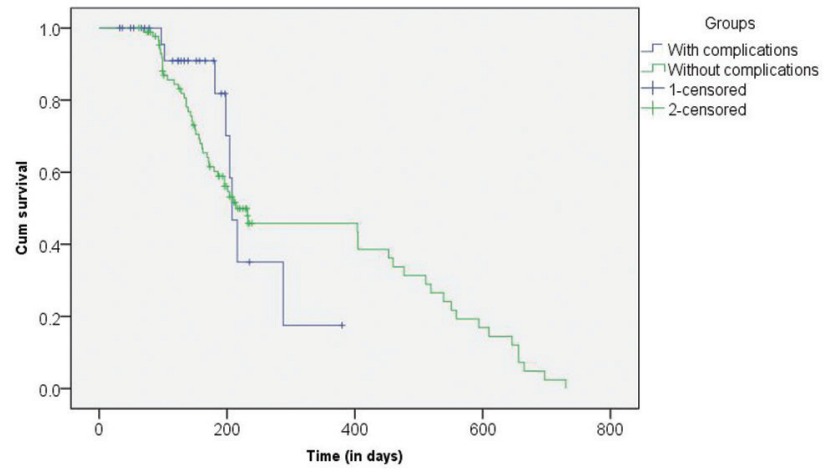

Fig. 7 Kaplan-Meier curve showing survival differences between percutaneous endoscopic gastrostomy with and without complications.
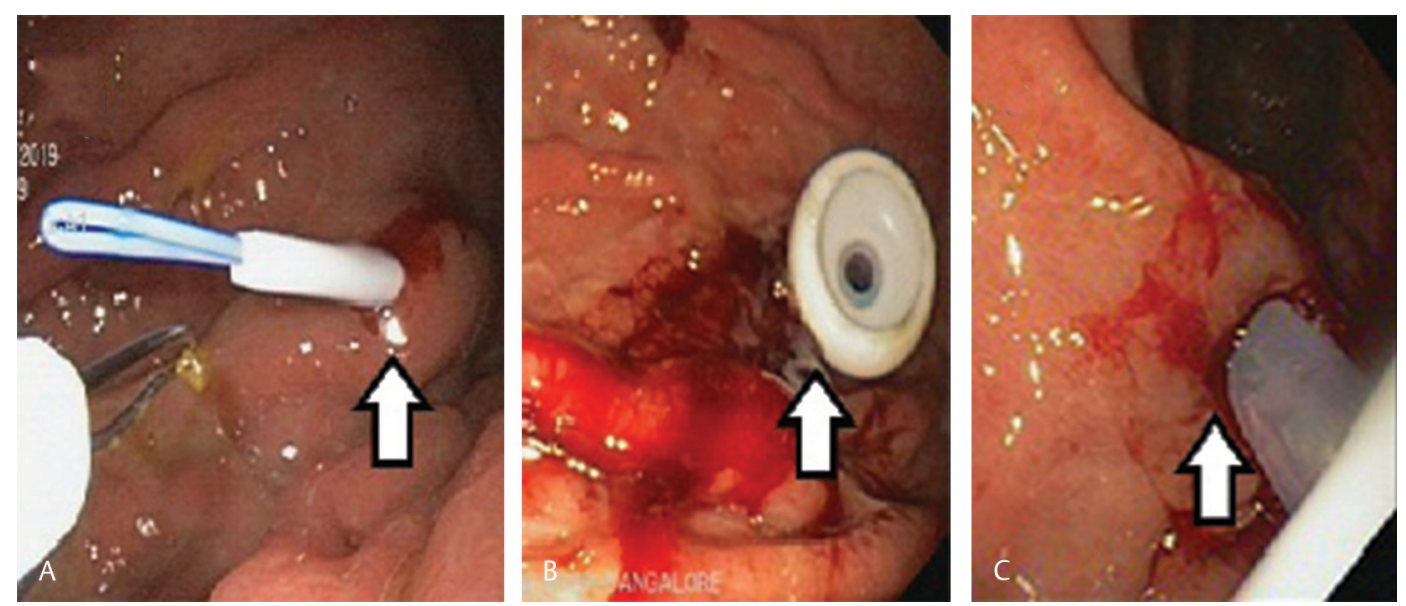

Fig. 6 (A) An inclined entry of angiocatheter that was responsible for percutaneous endoscopic gastrostomy (PEG) site bleeding (B) and (C) PEG site bleeding. 
with carbon dioxide or use of water soluble contrast media delineates stomach outline and is helpful in altered upper GI anatomy. ${ }^{15,16}$ A cross-table lateral fluoroscopy image can identify colonic interposition between the stomach and abdominal wall. ${ }^{30}$ Fluoroscopy-assisted colonoscopy can be used for moving transverse colon away from the anticipated PEG site in pseudo-obstruction (double-tube technique). ${ }^{31}$ PEG site chosen in F-PEG at lower one-third of body of stomach equidistant from greater and lesser curvature lateral to rectus muscle can avoid injury to epigastric or gastroepiploic arteries and might reduce bleeding complications. ${ }^{29}$ Placement of jejunal extension tubes (J-arm) through existing PEG tube can be performed with fluoroscopy that determines its exact position in the bowel (PEG-J). ${ }^{32}$

The adverse events after PEG in the literature range from 16 to $39 \%{ }^{24}$ The most common post-PEG complication reported is inadvertent PEG tube removal or dislodgement due to pliability of inner bolster. ${ }^{25}$ We suggest boxing hand wrap/use of abdominal binder for elderly patients and DIN to prevent tube dislodgement. ${ }^{33}$

BBS is an unusual complication with an estimated incidence ranging from 0.3 to $2.4 \%{ }^{34}$

In our series, BBS was noted in $12.7 \%$ and all of them underwent endoscopic replacement of PEG tube through the same site. BBS occurs due to inappropriate positioning of the gauge below the external bumper causing excessive pressure and tissue ischemia. ${ }^{35}$ BBS could have been prevented by appropriate positioning of the PEG tube, leaving a small distance between the skin and the external fixator with daily rotation of the gastrostomy tube by 180 to 360 degrees. ${ }^{18} \mathrm{BBS}$ associated with PEG site bleeding can be prevented by releasing the tightened bumpers after 48 hours. ${ }^{36}$ Clogged PEG tube is usually due to inadequate flushing with warm water before or after the PEG feed. ${ }^{36}$

In our study, PSI was noted in $11 \%$ with Klebsiella and Pseudomonas being the common organisms. In the study by Krishna et.al, PSI was noted in $24 \%$ with Pseudomonas being the most common organism. ${ }^{23}$ The major determinants of PSI are antibiotic prophylaxis, PEG insertion techniques, wound care practices, and debilitated patients. This study suggests the organism and sensitivity pattern of PSI infection to guide the infection prevention and treatment strategy. ${ }^{37}$ Of the PSI patients, eight patients had an infection in other sites.

A Cochrane systematic review showed pneumonia was significantly higher in patients with oropharyngeal dysphagia and favors PEG over NG in these patients. The occurrence of aspiration pneumonia depends on the cough reflex, volume and $\mathrm{pH}$ level of refluxed material, and the integrity of the immune system. ${ }^{3}$ We observed that DIN had higher association with development of aspiration pneumonia. The 6 -month mortality rate was $42 \%$, which was equivalent to the earlier reported mortality rates of 1.5 to $54 \%{ }^{38,39}$

The main strength of the study is availability and use of fluoroscopy during PEG procedure in unanticipated technically challenging situations; it may be difficult to transfer the sedated patient to a fluoroscopy room. The sample size was adequate with variety of indications and adverse events of PEG.
There are some drawbacks in the study. First, it being a retrospective single-center study has its inherent limitations. Second, aspiration pneumonia accounted for about one-fourth of mortality that is difficult to attribute as a complication of gastroesophageal reflux from feeds or due to underlying primary illness. Prospective multicenter studies with larger sample size will be required to validate our findings.

In conclusion, presence of fluoroscopy in the endoscopy theater will help in accurate PEG site (F-PEG) selection and placement. Age $>60$ years and DIN were independent risk factors associated with tube dislodgement, PSI, and development of buried bumper. The survival after PEG placement depends more on the primary disease than on the PEGrelated complications.

\section{Authorship Criteria}

Avinash Bhat Balekuduru was involved in the concept of study, drafting of article, revising it, and final approval. Shruti Sagar, Narendra M, Gajendra R, and Vinit KK were involved in acquisition of data or collection, drafting of the article, and final approval.

Satyaprakash Bonthala Subbaraj was involved in approval of the study.

\section{Funding}

No grants were received for the study.

\section{Conflict of Interests}

The authors declare that there is no conflict of interests regarding the publication of this paper.

\section{Acknowledgments}

The authors would like to thank Dr. Amit Kumar Dutta and Dr. Shivaraj Somanna for their excellent assistance as statistical reviewer. We would like to thank endoscopic staff-Maggie, Nagarathna, Padma, Jaya, Ranjan, Asha, Kiran, and Shilpa for compiling the data.

\section{References}

1 Gauderer MW, Ponsky JL, Izant RJ Jr. Gastrostomy without laparotomy: a percutaneous endoscopic technique. J Pediatr Surg 1980;15(6):872-875

2 Gordon C, Hewer RL, Wade DT. Dysphagia in acute stroke. $\mathrm{Br}$ Med J (Clin Res Ed) 1987;295(6595) :411-414

3 Gomes CA Jr, Lustosa SA, Matos D, Andriolo RB, Waisberg DR, Waisberg J. Percutaneous endoscopic gastrostomy versus nasogastric tube feeding for adults with swallowing disturbances. Cochrane Database Syst Rev 2010;11(11):CD008096

4 Moran BJ, Taylor MB, Johnson CD. Percutaneous endoscopic gastrostomy. Br J Surg 1990;77(8):858-862

5 Gauderer M. Twenty years of percutaneous endoscopic gastrostomy: origin and evolution of a concept and its expanded applications. Gastrointest Endosc 1999;50(6):879-883

6 DeLegge $\mathrm{MH}$. Enteral access and associated complications. Gastroenterol Clin North Am 2018;47(1):23-37

7 Prabhakaran S, Doraiswamy VA, Nagaraja V, et al. Nasoenteric tube complications. Scand J Surg 2012;101(3):147-155

8 Wicks C, Gimson A, Vlavianos P, et al. Assessment of the percutaneous endoscopic gastrostomy feeding tube as part of an integrated approach to enteral feeding. Gut 1992;33(5):613-616

9 Mellinger JD, Ponsky JL. Percutaneous endoscopic gastrostomy: state of the art, 1998. Endoscopy 1998;30(2):126-132 
10 Pearce CB, Duncan HD. Enteral feeding. Nasogastric, nasojejunal, percutaneous endoscopic gastrostomy, or jejunostomy: its indications and limitations. Postgrad Med J 2002;78(918):198-204

11 Niv Y, Abuksis G. Indications for percutaneous endoscopic gastrostomy insertion: ethical aspects. Dig Dis 2002;20(3-4):253-256

12 Hucl T, Spicak J. Complications of percutaneous endoscopic gastrostomy. Best Pract Res Clin Gastroenterol 2016;30(5):769-781

13 Onder A, Kapan M, Arikanoglu Z, et al. Percutaneous endoscopic gastrostomy: mortality and risk factors for survival. Gastroenterol Res 2012;5(1):21-27

14 Jain R, Maple JT, Anderson MA, et al; ASGE Standards of Practice Committee. The role of endoscopy in enteral feeding. Gastrointest Endosc 2011;74(1):7-12

15 Dobos S, Thill V, Deressa BK, et al. Gastrostomy placement: when fluoroscopy helps the endoscopist. Acta Gastroenterol Belg 2018;81(4):525-527

16 Zenitani M, Uehara S, Nara K, et al. Fluoroscopy-guided percutaneous endoscopic gastrostomy in children: a simple and safe technique. Surg Laparosc Endosc Percutan Tech 2016;26(2):167-170

17 Verma S, Dutta U. Percutaneous endoscopic gastrostomy: an effective yet underutilized procedure in India. Journal of Digestive Endoscopy 2019;10(3):155-157

18 Rahnemai-Azar AA, Rahnemaiazar AA, Naghshizadian R, Kurtz A, Farkas DT. Percutaneous endoscopic gastrostomy: indications, technique, complications and management. World J Gastroenterol 2014;20(24):7739-7751

19 Balekuduru A, Kumar A, Dutta AK, Aravind H, Subbaraj SB. Feasibility and safety assessment of home based gastrostomy tube feed - a tertiary care centre experience. Trop Gastroenterol 2018;39(2):83-86

20 Pih GY, Na HK, Hong SK, et al. Clinical outcomes of percutaneous endoscopic gastrostomy in the surgical intensive care unit. BMC Gastroenterol 2018;18(1):101

21 Cyrany J, Rejchrt S, Kopacova M, Bures J. Buried bumper syndrome: a complication of percutaneous endoscopic gastrostomy. World J Gastroenterol 2016;22(2):618-627

22 Jain NK, Larson DE, Schroeder KW, et al. Antibiotic prophylaxis for percutaneous endoscopic gastrostomy. A prospective, randomized, double-blind clinical trial. Ann Intern Med 1987;107(6):824-828

23 Krishna S, Singh S, Dinesh KR, Kp R, Siyad I, Karim S. Percutaneous endoscopic gastrostomy (PEG) site infections: a clinical and microbiological study from university teaching hospital, India. J Infect Prev 2015;16(3):113-116

24 Enestvedt BK, Jorgensen J, Sedlack RE, et al; ASGE Training Committee 2013-2014. Endoscopic approaches to enteral feeding and nutrition core curriculum. Gastrointest Endosc 2014;80(1):34-41
25 Shangab MO, Shaikh NA. Prediction of risk of adverse events related to percutaneous endoscopic gastrostomy: a retrospective study. Ann Gastroenterol 2019;32(5):469-475

26 Prosser B. Common issues in PEG tubes-what every fellow should know. Gastrointest Endosc 2006;64(6):970-972

27 Heyland DK. Nutritional support in the critically ill patients. A critical review of the evidence. Crit Care Clin 1998;14(3):423-440

28 Selim Youssef GY, Alnajjar A, Elsherbiny M. A comparison of percutaneous endoscopic gastrostomy and nasogastric tube feeding in prolonged dysphagic stroke patients. Adv Arab Acad Audio-Vestibul J 2015;2:14-18

29 Karthikumar B, Keshava SN, Moses V, Chiramel GK, Ahmed M, Mammen S. Percutaneous gastrostomy placement by intervention radiology: Techniques and outcome. Indian J Radiol Imaging 2018;28(2):225-231

30 Shukla PA, Kolber MK, Tapnio R, Zybulewski A, Kumar A, Patel RI. Safety and feasibility of ultrasound-guided gastric access for percutaneous transabdominal gastrostomy tube placement. Gastroenterol Res 2019;12(3):115-119

31 Fukita Y, Katakura Y, Adachi S, et al. Colonoscopy-assisted percutaneous endoscopic gastrostomy to avoid a gastrocolocutaneous fistula of the transverse colon. Endoscopy 2014;46(Suppl 1 UCTN) :E60

32 Uflacker A, Qiao Y, Easley G. Patrie J, Lambert D, de Lange EE. Fluoroscopy-guided jejunal extension tube placement through existing gastrostomy tubes: analysis of 391 procedures. Diagn Interv Radiol 2015;21(6):488-493

33 Gupta A, Singh AK, Goel D, Gaind AN, Mittal S. Percutaneous endoscopic gastrostomy tube placement: a single center experience. Journal of Digestive Endoscopy 2019;10(3):150-154

34 Geer W, Jeanmonod R. Early presentation of buried bumper syndrome. West J Emerg Med 2013;14(5):421-423

35 Libânio D, Pimentel-Nunes P. Early buried bumper syndrome - to leave or not to leave. GE Port J Gastroenterol 2018;25(3):115-116

36 Naik RP, Joshipura VP, Patel NR, Haribhakti SP. Complications of PEG-prevention and management. Trop Gastroenterol 2009;30(4):186-194

37 Jafri NS, Mahid SS, Minor KS, Idstein SR, Hornung CA, Galandiuk S. Meta-analysis: antibiotic prophylaxis to prevent peristomal infection following percutaneous endoscopic gastrostomy. Aliment Pharmacol Ther 2007;25(6):647-656

38 Sanders DS, Carter MJ, D'Silva J, James G, Bolton RP, Bardhan KD. Survival analysis in percutaneous endoscopic gastrostomy feeding: a worse outcome in patients with dementia. Am J Gastroenterol 2000;95(6):1472-1475

39 Varnier A, Iona L, Dominutti MC, et al. Percutaneous endoscopic gastrostomy: complications in the short and long-term follow-up and efficacy on nutritional status. Eura Medicophys 2006;42(1):23-26 